\title{
Cultural relaxation methods for minor mental health disorders
}

\author{
Daya Somasundaram
}

Summary

Traditional techniques used to produce relaxation can promote and maintain wellbeing in healthy individuals too. They can prevent illnesses including minor mental health disorders from developing in those faced with psychosocial problems by countering stress, states of tension and improving coping capacities. Further, they can cure a variety of minor mental health disorders that have already developed. In cases of psychosomatic diseases like hypertension, gastritis, bronchial asthma and eczema, they may reduce the need for medication. In the ubiquitous somatisation found in all the various health care settings, traditional relaxation techniques can be helpful. The saving to health care services, benefit to the community and relief for the individual patient can be considerable.

SL J Psychiatry 2012; 3 (2) 3-6

\section{Introduction}

Around the world, the incidence and prevalence of minor mental health disorders in the general population is high. Around $10-20 \%$ of the population is believed to suffer from minor mental health disorders ranging from anxiety and minor depression to somatoform disorders (1). Worldwide it has been established that from a quarter up to a third of patients seeking help at primary health care facilities may be suffering from different types of psychological disorders $(2,3,45,6)$. A further proportion may be having various psychosocial problems manifesting through physical symptoms. These patients usually present with physical symptoms that do not have an organic basis; or, are not explainable by any organic condition that is present. There may be coexistent anxiety, depression, or sexual problems. Earlier referred to as conversion hysteria, functional disorders, hypochondriasis, psychogenic pain, neurasthenia, hyperventilation syndrome and Briquet's syndrome, they have now been brought together under the rubric of somatoform disorder or somatisation.

Somatisation may be commoner in developing countries where it is said that mental distress is often experienced and expressed in somatic terms (7). Somatisation could also present to more specialized services such as medicine, surgery, gynecology, dermatology, dentistry, paediatrics and rheumatology. Although doctors maybe aware of the core problem, it is rarely identified or managed appropriately. The cost to the patient, family, society and to the health services is considerable (2). As western medicine has not evolved effective or satisfying treatment for these conditions, patients seek help from traditional sectors.

Further, many common physical diseases are caused or exacerbated by psychosocial factors. Apart from the well-known psychosomatic diseases like bronchial asthma, gastritis, hypertension and eczema, the list of physical illnesses that are linked to stress is growing and include coronary heart disease, rheumatoid arthritis, diabetes, migraine, fibromyalgia, chronic fatigue syndrome, ulcerative colitis and irritable bowel syndrome.

The recommended first line treatment in the West for individuals with minor mental health disorders due to psychosocial problems is counseling or psychotherapy and more recently, Cognitive Behavioural Therapy (CBT). Yet in actual practice, they are managed with psychotropic medications that could include antidepressants, anti-anxiety agents, sedatives, hypnotics and anti-psychotics. But despite claims by pharmaceutical companies with evidence from randomized controlled trials, the effectiveness of drugs compared to placebo is equivocal $(8,9)$. In the longterm, drugs, with their side-effects and abuse potential, may do more harm than good. Also, those in nonwestern cultures may find modern psychiatric methods of treatment such as psychotherapy unacceptable. Thus it is not unexpected that the outcome of counseling is poor in non-western contexts. Further, the very assumptions on which psychotherapeutic interventions are based may be culturally flawed and thus the issues one takes up in psychotherapy will be erroneous.

Stress leads to many physiological changes in the body (particularly activation of the sympathetic division of the autonomic nervous system and the adrenal medulla and cortex of the endocrinal system) that in turn, may be lead to a host of physical diseases. The lifesaving role of the hypothalmo -adrenal cortical axis to secrete corticosteroids under stress was first recognized by Selye who described the exhaustion of the General Adaptation Syndrome under extreme situations (10). In trauma, on the other hand, there is a chronic state of hyperactivation of this system leading to hyperarousal. Western biomedicine realised that one of the best ways to relieve stress is through relaxation exercises. Jacobson first introduced relaxation methods into Western medicine (11). Jacobson's exercises produced a state of relaxation which was hypothesized to be incompatible with a state of tension due to stress. 
When a person is under stress or emotionally disturbed, the breathing becomes rapid, irregular and shallow. This type of rapid over breathing, sometimes called hyperventilation syndrome in extreme cases, leads to excessive carbon dioxide being expelled through the lungs with resultant changes in blood acid base balance and a drop in serum calcium (12). This manifests in medically well-known signs and symptoms of hypocalcemia. On the contrary, when a person is relaxed, the breathing is calm, deep and regular. One of the basic methods of Jacobson is deep breathing exercises. The goal in therapy is to bring breathing under conscious control and make it deep, smooth and regular. This usually entails learning abdominal, diaphragmatic breathing in contrast to the usual thoracic type of breathing.

Another marked sign of stress is tension in the body. All the muscles become tense. Pain in the neck muscles and tension headache are common symptoms following stress. Further, prolonged and abnormal contraction of muscles can lead to ligamentous strains and arthritis in the joints. Jacobson's progressive muscular relaxation technique counters this state of tension by gradually producing relaxation in each group of muscles in turn. Research by Benson and others have shown that repetition of a sound can produce relaxation and beneficial changes in physiological parameters such as pulse, blood pressure, breathing, skin sweating, muscular tension and brain electrical activity (13). Benson called it the 'relaxation response'. This is the basis of the popular Transcendental Meditation (TM).

Traditional methods, physiologically similar to Jacobson's techniques, have been used for centuries in Asia. The Yogic pranayamam and Buddhist form of mindful breathing (Ana Pana Sati) are culturally well known breathing exercises that produce conscious, deep, regular abdominal (diaphragmatic) breathing. The Yogic Shanthi or Sava Asana produces progressive muscular relaxation. Similar to Benson's relaxation response, the Hindu Jappa, Catholic Rosary or prayer beads, Islamic Dhikir, Buddhist Pirith or chanting are culturally familiar and acceptable. For meditation, Dhyanam, Samadhi, Vipassana, Contemplation and other methods are in use. In addition, traditional oil massage used in Ayurvedic and Siddha medicine can induce states of relaxation.

A worldwide panel of trauma experts on the treatment of those exposed to disaster and mass violence identified that producing a sense of calming as an important empirically supported intervention principle to counteract the increase in emotionality, hyperarousal, anxiety, distress, fear and avoidance found in these post traumatic situations (14). They recommend deep, diaphragmatic breathing; deep muscle relaxation; yoga; and mindfulness techniques drawn from Asian culture and meditation methods.

Studies report that psychosomatic diseases like asthma, gastritis, hypertension, eczema as well as non-insulin dependent diabetes mellitus and other common ailments will benefit from traditional relaxation methods (15, $16,17,18,19)$. However, not all studies have shown uniform benefit (20).
Mindfulness-based therapeutic interventions appear to be effective in the treatment of recurrent depression, anxiety, Post-Traumatic Stress Disorder (PTSD), psychosis, borderline personality disorder, self-harm behaviour and in reducing substance use and recidivism rates in incarcerated population $(21,22,23,24)$. A randomized comparison of CBT based Narrative Exposure Therapy (NET) to relaxation-meditation for PTSD in Sri Lanka, showed that there was no significant difference between the two (25).

\section{Cultural Relaxation methods}

Four basic methods that are physiologically similar to Jacobson's techniques are discussed.

\section{Breathing exercises}

The goal of the exercises is to bring breathing under conscious control. Clients are taught to observe their breathing as it flows in and out, to breathe slowly, continuously and deeply. They are taught to concentrate on the abdomen slowly expanding while they draw in their breath deeply. A 'mantra' or word is said while breathing in and out. Progressively, over months, the time taken to say the word is increased. Alternatively, when the above method is difficult, the person is taught to count slowly up to three, while breathing in and again while breathing out. The number is then gradually increased.

\section{Progressive Muscular Relaxation}

The aim is to gradually relax each part of the body, step by step, until the whole body is relaxed. Subjects are made to lie down flat, facing up, on a mat or bed. They are then taught to first concentrate their mind on their toes and while relaxing and then move up their body - toes, soles, ankle, calf, knee, thigh, sexual organs, anus, abdomen, back, chest, fingers, hands, elbow, arms, shoulders, neck, face and scalp. They are asked to recognise a sense of tranquility in each part until at the end, the whole body is deeply relaxed.

\section{Regular Repetition of Words}

Repetition of a holy verse, God's name, a mantra, meaningful word, idiom or saying is used. Initially, clients are asked to repeat or recite the word or saying for five minutes or longer two times a day at a convenient time and place, when and where there will be no disturbance, in a comfortable posture in a voice that is not too loud. They are asked to continue this practice for about two weeks or longer until the recitation becomes easy and the articulation and posture becomes effortless. Then they are asked to make the voice softer. They are then asked to say it without a sound, with only the muscles of articulation involved. The next stage is to just repeat it in the mind and think of it. At some point in the process, when the client is judged to have developed some mastery, they are asked to practice at other times as necessary, such as when they are feeling anxious.

\section{Meditation}

At first the patient is asked to choose an object for meditation. The person's deity, God, respected saint or elder or a beautiful natural scene was often chosen 
as the subject of meditation. Alternatively, the above mentioned word or phrase was used. In patients who found this difficult, they were asked to observe the breath (in flow and out flow of breathing at the tip of the nose or the rising or falling of the abdomen). They were taught to sit in a posture that is comfortable. They were then asked to concentrate the mind and keep it continuously on the chosen object. They were taught to focus the mind on the chosen object.

\section{Discussion}

Therapy with Relaxation methods being nonpharmacological is safe, non-toxic with no side effects and cheap. Further, in developing countries such as in Sri Lanka where resources are scarce, traditional methods are useful in helping those with psychosocial problems. However, awareness about these methods or those adept in its use is low.

A revival of the traditional knowledge systems and practices together with respect and interest for them is needed. Western medicine can play an important role by lending its considerable social respectability towards establishing legitimacy and attraction. Recently there has been renewed interest in these practices particularly in meditation and yoga. The influence of western practitioners and sympathizers can be seen in these efforts. Moreover, the experiences of using Cultural Relaxation Therapies (CRT) in diverse settings such as Sri Lanka, Cambodia and Australia suggest that this form of therapy could become a vital component in a multi-disciplinary approach and gain wider acceptance within the 'western' model of treatment $(26,27)$.

Being culturally accepted, traditional methods work within the belief systems of the populations. Thus, it is not necessary to impose western psychiatric nosology or explanatory systems and the 'category fallacy' can be avoided (28). By not giving drugs, no harm is done in the majority of minor mental health disorders. If they can develop some faith in their practice much benefit can be accrued (29). The responsibility for their condition is subtly shifted to the person themselves, and they are taught to take control of their bodily and mental processes. The advantages of the traditional methods described is that they work at the physical, psychological, social and spiritual levels at the same time producing an integrated holistic effect.

\section{Physical Level}

The first two exercises are directed at bodily processes. The third method of repetition of words is also initially a very physical method of using the vocal chords that produces the 'relaxation response' with muscular relaxation and beneficial changes in physiological parameters such as heart rate, breathing, blood pressure, brain electrical activity, sweating and cellular metabolism (13). As mental distress in Asian cultures is often experienced and expressed in somatic terms, it will benefit from interventions structured initially in physical terms (7). These practices are especially helpful for somatoform disorders such as somatisation and hypochondriasis and conversion states where there is abnormal preoccupation with the body. The exercises will help to divert the attention away into more healthy body awareness. Anxiety, depression, PTSD and culture bound syndromes that manifest through somatic complaints will also be helped by these practices.

\section{Psychological Level}

Mentally, these methods produce a state of calming countering states of arousal and anxiety. They are indicated in minor mental health disorders like anxiety disorder, panic disorder, phobias, PTSD and hypochondriasis. Relaxation exercises are used as part of other interventions, for example in the behavioural method of systematic desensitization.

\section{Social level}

Socially, it is becoming evident that physical symptoms, the experience of these symptoms or illness

itself, health seeking behaviour, and explanation and beliefs about causation are culturally determined (26). Social processes may actually influence the pathophysiology to change bodily processes. Thus a therapy that works through culturally accepted practices and beliefs systems could be of immense benefit. It may be able to use or reverse the very psychosocial processes that were responsible for the original problem or complaint. But more importantly, traditional methods that co-opt cultural processes can be used to address widespread problems that have taken on a social dimension.

\section{Spiritual level}

When methods are culturally familiar, they tap into past childhood, community and religious roots and thus release a rich source of associations that can be helpful in therapy and the healing process. Mindfulness and meditation draw upon hidden resources within the individual and open into dimensions that can create spiritual well-being and give meaning to what has happened. Although these techniques do no formal psychotherapy, they may accomplish what psychotherapy attempts to do by releasing cultural and spiritual restorative processes.

\section{Conclusion}

Traditional techniques used to produce relaxation can promote and maintain wellbeing in healthy individuals too. They can prevent illnesses including minor mental health disorders from developing in those faced with psychosocial problems by countering stress, states of tension and improving coping capacities. Further, they can cure a variety of minor mental health disorders that have already developed. In cases of psychosomatic diseases like hypertension, gastritis, bronchial asthma and eczema, they may reduce the need for medication. In the ubiquitous somatisation found in all the various health care settings, traditional relaxation techniques can be helpful. The saving to health care services, benefit to the community and relief for the individual patient can be considerable. 


\section{Daya Somasundaram}

Professor of Psychiatry

University of Jaffna, Sri Lanka

E mail: dayanandan.somasundaram@adelaide. edu.au

\section{References}

1. Goldberg P, Huxley P. Common Mental Disorders. London: Routledge. 1992.

2. Samarasinghe D. Recognition and management of functional complaints. Ceylon Medical J 1991; 36: 23 26.

3. Goldberg D., \& Blackwell B. Psychiatric illness in general practice: a detailed study using a new method of case identification. British Medical J 1970; 2: 439-443. CrossRef

4. Nikapota A, Patrick V, Fernando L. Aspects of psychiatric morbidity in the out-patient population of a general hospital in Sri Lanka. Indian J of Psychiatry 1981; 23: 219-223.

5. Bridges K, Goldberg, D. Somatic presentation of DSM III psychiatric disorders in primary care. $\mathrm{J}$ of Psychosomatic Research 1987; 29: p 563. CrossRef

6. Somasundaram, D. War Trauma and Psychosocial Problems: Out Patient Attendees in Jaffna. International Medical J 2001; 8: 193 - 197.

7. Bracken, PJ, Geiller, JE, Summerfield, D. Psychological Responses to War and Atrocity: The Limitations of Current Concepts. Social Science and Medicine 1995; 40:1073-1082.CrossRef

8. Kirsch I, Moore T, Scoboria A, Nicholls SS. The emperor's new drugs-An analysis of antidepressant medication data submitted to the U.S. Food and Drug Administration. Prevention \& Treatment 2002; 5: 1- 11 CrossRef

9. Parker G, Anderson, IM, Haddad, P. Antidepressants on trial: how valid is the evidence? British J of Psychiatry. 2009; 194: 1-3.CrossRef

10. Selye, H. The stress of life. New York: McGraw Hill. 1978.

11. Jacobson, E. Progressive Relaxation. Chicago: Chicago University Press. 1938.

12. Magarian G. Hyperventilation syndrome. Medicine 1982; 61, 219-233. CrossRef

13. Benson, H. The Relaxation Response. New York: Avon Books. 1975.

14. Hobfoll, SE, Watson P et al. Five Essential Elements of Immediate and Mid-Term Mass Trauma Intervention:
Empirical Evidence. Psychiatry 2007; 70:(4), 283-315. CrossRe

15. Nagendra, HR, Nagarathna, R. A New Light for Asthmatics. Bangalore: Vivekananda Kendra Yoctas. 1986.

16. Nagendra, HR, Nagarathna, R. New Perspectives in Stress Management. KanniyaKumari: Vivekananda Kendra Yoga Research Foundation. 1988.

17. Patel, $\mathrm{CH}$. Yoga and Biofeedback in the management of Hypertension. The Lancet, 1973; 302: 1053-1055. CrossRef

18. Nagendra, HR, Nagarathna R. Further Studies on Diabetes Mellitus. Bangalore: Vivekananda Kendra Yoga Therapy \& Research Centre. 1984.

19. Monro, R, Nagarathna, R, Nagendra, HR. Yoga for common ailments. London: Simon and Schuster Inc. 1990.

20. Van Montfrans, GA, Karemaker, JM, Wieling, W. Relaxation therapy and continuous ambulatory blood pressure in mild hypertension: a controlled study. British Medical J. 1990; 300: 1368-1372. CrossRef

21. Baer, R. Mindfulness- Based Treatment Approaches. Burlington, Academic Press. 2006.

22. Ivanovski, B, Malhi, GS. The psychological and neurophysiological concomitants of mindfulness forms of meditation. Acta Neuropsychiatrica 2007; 19: 76-91. CrossRef

23. Miller, JJ, Fletcher, K, Kabat-Zinn, J. Three-year followup and clinical implications of a mindfulness meditationbased stress reduction intervention in the treatment of anxiety disorders. General Hospital Psychiatry. General Hospital Psychiatry 1995; 17:192-200. CrossRef

24. Somasundaram, DJ Treatment of Massive Trauma. Advances in Psychiatric Treatment 1997; 3: 321-331. CrossRef

25. Catani, C, Kohiladevy, M, Ruf, M, Schauer, E, Elbert, T, Neuner, F. Treating children traumatized by war and Tsunami: A comparison between exposure therapy and meditation-relaxation in North East Sri Lanka. BMC Psychiatry 2009; 9: 22. CrossRef

26. Somasundaram, D. Using Traditional Relaxation Techniques in Health Care. Community Mental Health and Wellbeing Bulletin 2010; 3: 1, 14-30.

27. Somasundaram, D Using cultural relaxation methods in post-trauma care among refugees in Australia, International Journal of Culture and Mental Health 2010; 3: 1, 16-24. CrossRef

28. Kleinman, AM. Patients and Healers in the Context of Culture. Berkeley: University of California Press. 1980.

29. Benson, H, Stark, M. Timeless Healing: The Power and Biology of Belief. New York: Scribner. 1996. 